\title{
Multi-Degree of Freedom Tuning Fork Gyroscope Demonstrating Shock Rejection
}

\author{
Adam R. Schofield, Alexander A. Trusov, and Andrei M. Shkel \\ MicroSystems Laboratory \\ Department of Mechanical and Aerospace Engineering \\ University of California, Irvine, Irvine, CA, USA \\ \{adam.schofield, atrusov, ashkel\}@uci.edu
}

\begin{abstract}
This paper presents a z-axis MEMS tuning fork rate gyroscope with multi-degree of freedom (DOF) sense modes designed to provide structural robustness to environmental drifts. This concept combines the temperature robustness of multiDOF sense modes with the common mode rejection capabilities of tuning fork architectures. The device consists of an antiphase actuated 2-DOF drive mode where each drive mass contains a 2-DOF sense mode, thus forming an overall 6-DOF mechanical system. The flat gain region of the 2 -DOF sense mode provides immunity to both environmental variations and fabrication imperfections, while the anti-phase forcing of the drive mode induces an anti-phase Coriolis response allowing for the cancellation of common mode inputs such as ambient vibrations. Impulse responses were used to characterize the effect of acceleration loads on the device where a differential signal resulted in $14 \mathrm{~dB}$ of reduction in amplitude versus a single output.
\end{abstract}

\section{INTRODUCTION}

For many applications, gyroscopes are subject to a wide variety of changing environmental conditions such as temperature, pressure, and ambient vibrations. The robustness of the sensor to these external influences during operation is critical for adequate performance. A level of robustness is commonly achieved through electronic control systems, such as a temperature compensation circuit which post-processes the output of the mechanical sensor depending upon temperature or an automatic mode matching controller [1], [2]. Robustness to ambient vibrations, however, is generally addressed by the mechanical design through the use of tuning fork driving architectures. Tuning fork designs have the ability to reject common mode inputs due to anti-phase forcing which results in anti-phase Coriolis responses [3], [4].

Previously, we introduced a gyroscope concept to address the challenges of temperature dependent drifts by means of mechanical design based upon increasing the sense mode degrees of freedom (DOF) [2]. The gyroscope structure consists of a 1-DOF drive and 2-DOF sense mode (3-DOF overall) and was shown to be robust to temperature changes due to the constant gain region in the sense mode frequency response. In order to address issues associated with ambient vibrations, we introduced a multi-DOF tuning fork concept consisting of two 3-DOF gyroscopes coupled in the drive mode [5]. This new design combined the temperature robustness of multiDOF sense modes with the common mode rejection of tuning forks.

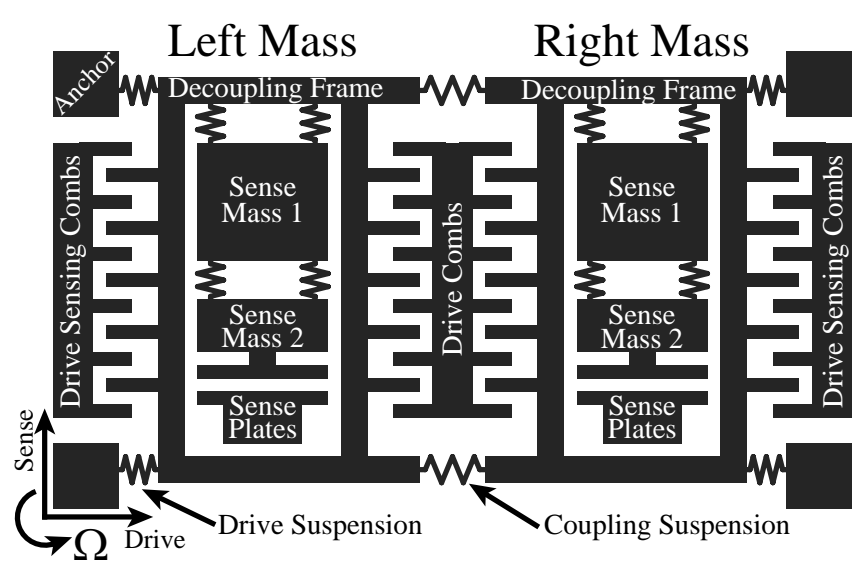

Fig. 1. Schematic of the multi-degree of freedom tuning fork gyroscope

This work focuses on the experimental demonstration of the common mode rejection capabilities of the multi-DOF tuning fork (MDTF) MEMS rate gyroscope. The design concept and its advantages are discussed in Section II. Section III describes the fabrication process used for building prototypes. Section IV presents the experimental characterization of the MDTF gyroscope. Impulse responses of the Coriolis accelerometers are analyzed in Section IV-A. The constant angular rate response curves for both right and left masses are presented in Section IV-B. Finally, the effect of mechanical shock on the Coriolis demodulation at zero rate is discussed in Section IV-C and Section V concludes the paper.

\section{DESIGN CONCEPT}

A schematic of the MDTF z-axis gyroscope concept is shown in Fig. 1. The design consists of two identical systems, labeled left mass and right mass, which are connected via a central suspension forming a 2-DOF coupled drive mode. The sense modes are also 2-DOF systems, so that the entire device forms a 6-DOF dynamic system. The ideal, linear time invariant (LTI) equations of motion are given by

$$
\begin{array}{r}
\left(m_{f}+m_{1}+m_{2}\right) \ddot{x}_{(L, R)}+c_{d_{(L, R)}} \dot{x}_{(L, R)}+k_{d} x_{(L, R)} \\
-k_{c} x_{(R, L)}=F_{d} \\
m_{1} \ddot{y}_{1_{(L, R)}}+c_{1_{(L, R)}} \dot{y}_{1_{(L, R)}}+\left(k_{1}+k_{2}\right) y_{1_{(L, R)}}
\end{array}
$$



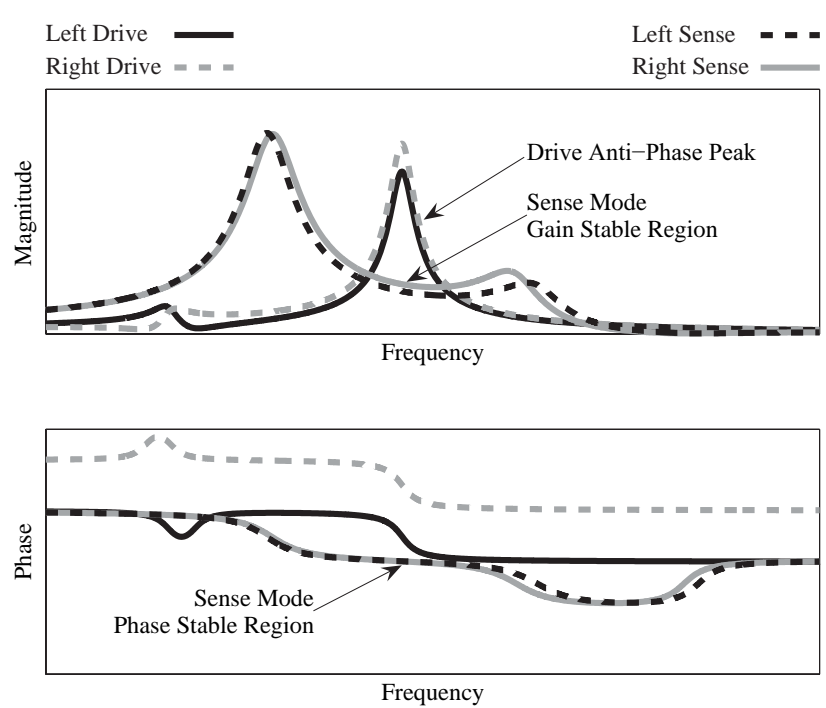

Fig. 2. Frequency responses of the MDTF drive and sense modes

$$
\begin{array}{r}
-k_{2} y_{2_{(L, R)}}=-2 \Omega_{z} \dot{x}_{(L, R)} \\
m_{2} \ddot{y}_{2_{(L, R)}}+c_{2_{(L, R)}} \dot{y}_{2_{(L, R)}}+k_{2} y_{2_{(L, R)}} \\
-k_{2} y_{1_{(L, R)}}=-2 \Omega_{z} \dot{x}_{(L, R)}
\end{array}
$$

where the subscript $(L, R)$ denotes left or right system respectively, $x$ is the displacement of the decoupling frame and sense masses moving together in the drive direction, $y_{1,2}$ are the displacements of sense mass 1 and 2 in the sense direction, $m_{f, 1,2}$ are the masses of the decoupling frames, sense mass 1 , and sense mass $2, c_{d, 1,2}$ are the total damping coefficients between the substrate and the drive mass, sense mass 1 , and sense mass 2 respectively, $k_{d}$ is the drive mode stiffness, $k_{c}$ is the drive mode coupling stiffness, $k_{1}$ is the stiffness between the frame and sense mass $1, k_{2}$ is the stiffness between sense mass 1 and sense mass $2, F_{d}$ is the applied driving force, and $\Omega_{z}$ is the angular rate.

The frequency response of a non-ideal MDTF is illustrated in Fig. 2. The drive system has two resonant modes: an inphase mode and a higher frequency anti-phase mode. Mechanically connecting the left and right masses via the coupling suspension ensures that the anti-phase operational mode occurs at the same frequency even in the presence of fabrication imperfections. Driving an ideally designed symmetric device in anti-phase would eliminate the in-phase response; however, as shown in Fig. 2, asymmetries due to imperfections will cause some motion at the lower resonant frequency.

The right and left sense modes are both 2-DOF systems and the resonant frequencies of each subsystem are designed to be symmetric relative to the drive mode anti-phase peak as shown in Fig. 2. The coupled sense systems have a broad region in the frequency response which has nearly constant gain and phase; operating the device in this region provides structural robustness to environmental drifts even in the presence of fabrication imperfections. The anti-phase motion of the drive

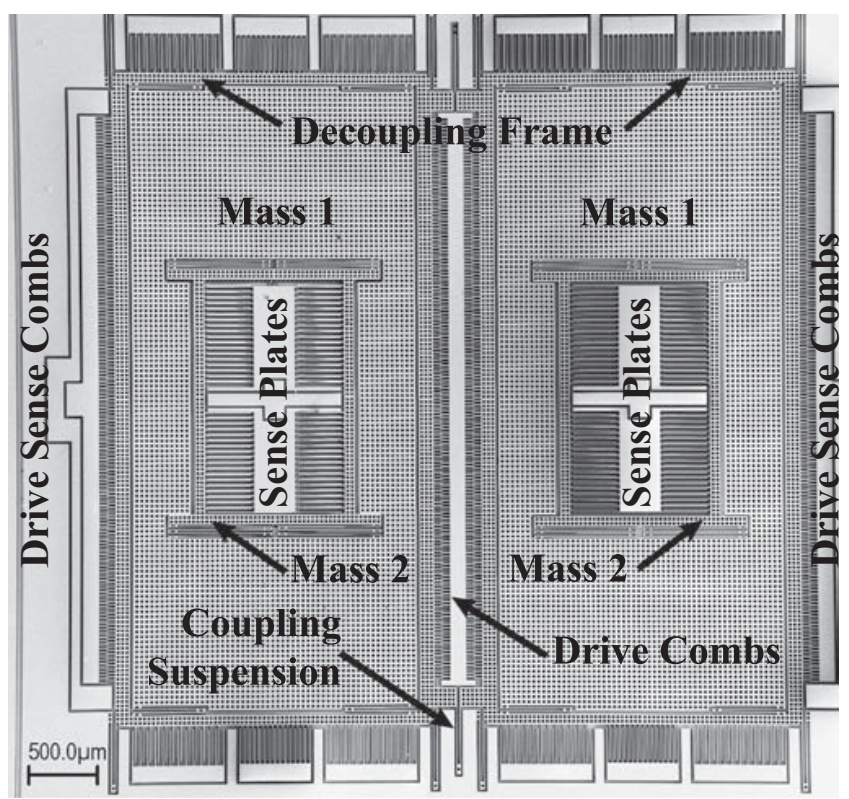

Fig. 3. SEM image of a fabricated multi-degree of freedom tuning fork gyroscope

TABLE I

PARAMETERS OF THE CHARACTERIZED DEVICE

\begin{tabular}{|l|c|}
\hline Parameter & Value \\
\hline \multicolumn{2}{|c|}{ Drive mode } \\
\hline In-phase natural frequency, kHz & 1.442 \\
\hline Anti-phase natural frequency, $\mathrm{kHz}$ & 2.167 \\
\hline Decoupling frame mass, kg & $1.90 \mathrm{e}-7$ \\
\hline Quality factor in air & $\approx 300$ \\
\hline Single-sided drive capacitance, $\mathrm{pF}$ & 0.27 \\
\hline \multicolumn{2}{|c|}{ Sense mode } \\
\hline Lower frequency, kHz & 1.8 \\
\hline Higher frequency, kHz & 2.4 \\
\hline Mass 1, kg & $7.07 \mathrm{e}-7$ \\
\hline Mass 2, kg & $8.29 \mathrm{e}-8$ \\
\hline Effective quality factor & $\approx 40$ \\
\hline Single-sided capacitance, $\mathrm{pF}$ & 0.11 \\
\hline
\end{tabular}

mode ensures that induced Coriolis responses will be in antiphase leading to differential rejection of common mode inputs.

\section{FABRICATION}

Prototypes of the multi-DOF tuning fork gyroscope were fabricated using an in-house wafer level SOI process with a $50 \mu m$ conductive device layer and $5 \mu m$ buried oxide layer. AZ photo-resist was lithographically defined using a Karl Suss MA6 exposure system. After photo-resist development, the wafers were subjected to a Deep Reactive Ion Etching (DRIE) step using a Surface Technology Systems (STS) Advanced Silicon Etching (ASE) tool. The minimum feature size used to define the capacitive gaps of the device was $5 \mu \mathrm{m}$. A timed HF 

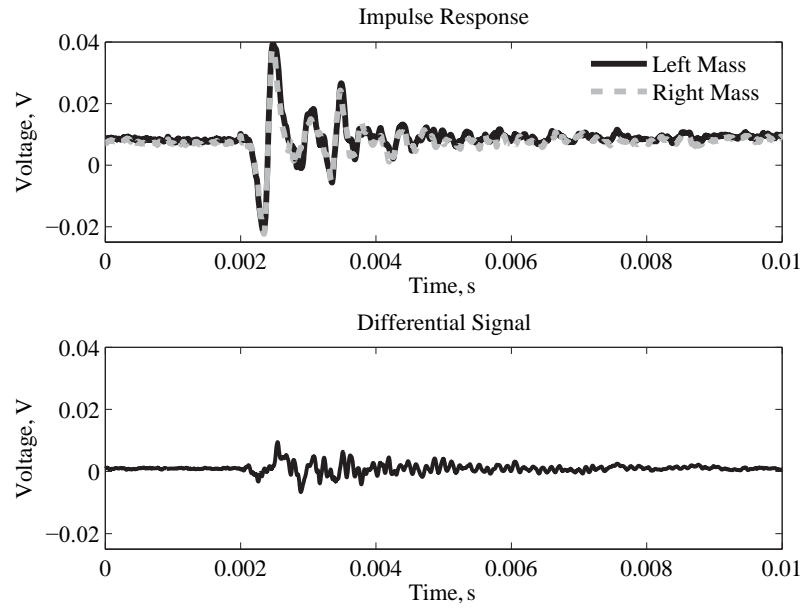

Fig. 4. Experimentally obtained time history of right and left sense mode output due to shock input

etch was then used to release the perforated mobile structures. The device was packaged in a 24-pin ceramic DIP package and wire-bonded.

Fig. 3 shows an SEM image of a fabricated multi-DOF tuning fork gyroscope. A central lateral comb drive is used to provide anti-phase drive actuation at the anti-phase resonant frequency to both the right and left drive masses simultaneously. The design uses mechanical mode decoupling between the drive and sense modes, where the sense masses are suspended relative to the decoupling frames. Each sense system consists of two masses, a larger mass (Mass 1) and a smaller mass (Mass 2) which has differential parallel plate capacitors for Coriolis detection.

Table I summarizes parameters of the device used for the experimental demonstration presented below. The fabricated 2DOF drive mode oscillator had an in-phase resonant frequency of $1.442 \mathrm{kHz}$ and an anti-phase frequency of $2.167 \mathrm{kHz}$, while both 2-DOF sense modes had resonant frequencies of approximately $1.8 \mathrm{kHz}$ and $2.4 \mathrm{kHz}$ which are still symmetric relative to the anti-phase peak in the presence of fabrication imperfections.

\section{EXPERIMENTAL RESULTS}

\section{A. Common Mode Rejection}

The response of both sense systems of the multi-DOF tuning fork gyroscope was characterized when subject to a shock input at atmospheric pressure. No driving voltages were applied to the device so that each sense mode system acted as a passive accelerometer. A constant DC probing bias of $10 \mathrm{~V}$ was applied to the gyroscope mass while each of the motional sense currents were sent to trans-impedance amplifiers with $100 k \Omega$ gain. The output voltages were captured using a LeCroy WaveSurfer Model 452 Digital Oscilloscope.

Fig. 4 (top) shows the experimentally obtained time histories of each sense mode system due to a mechanical impulse triggered at 0.002 seconds along the sense direction. The
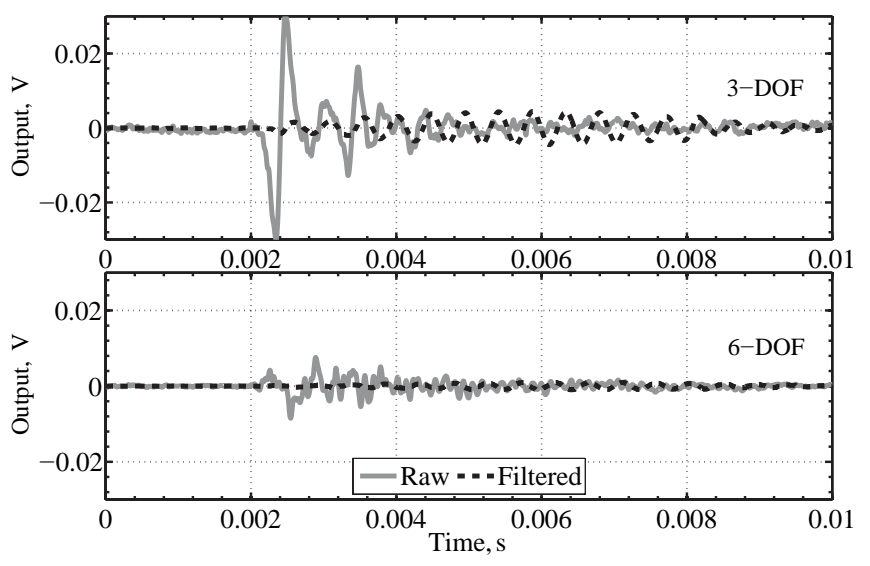

Fig. 5. Filtered time response of single mass (3-DOF) and differential signal (6-DOF)

output of each sense system responded in a common mode as expected. The difference of the two signals is shown in Fig. 4 (bottom) which results in a $12 \mathrm{~dB}$ reduction in amplitude versus the individual signals.

The impulse data was post-processed to model the effect of filtering during Coriolis demodulation. A band-pass filter with a $50 \mathrm{~Hz}$ bandwidth centered about the $2.167 \mathrm{kHz}$ drive frequency was used to filter a single sense mass and the differential signal representing the outputs of a 3-DOF and MDTF, respectively. The filtered 3-DOF signal shown in Fig. 5 (top) results in a $16 \mathrm{~dB}$ reduction of amplitude at the cost of time delay. Fig. 5 (bottom) shows the effect of bandpass filtering the differential signal; it results in an additional suppression of $18 \mathrm{~dB}$ due to the tuning fork operation.

\section{B. Coriolis Response}

The multi-DOF tuning fork gyroscope prototype was characterized under constant angular rate inputs to determine the individual scale factors. This was done for the individual masses to verify the anti-phase nature of their Coriolis responses; the final device output would be the differential of these two signals. For each output, a two stage demodulation of the electromechanical amplitude modulated (EAM) pickup signal was used to extract the Coriolis motion [2]. Two Advanced Measurement Technology Model 7265 lock-in amplifiers were used to generate the drive and carrier voltages as well as to demodulate the signals. The drive voltage of 3.535 Vrms AC at $2.167 \mathrm{kHz}$ combined with a $40 \mathrm{~V}$ DC bias was applied to the central lateral comb drive, while an AC carrier voltage of $3 \mathrm{Vrms}$ at $18 \mathrm{kHz}$ was applied to gyroscope mass. The device was mounted on an Ideal Aerosmith 1291BR rate table at room temperate and atmospheric pressure and tested for constant angular rates in the range of $\pm 60 \mathrm{deg} / \mathrm{s}$ (a range of $\pm 300 \mathrm{deg} / \mathrm{s}$ is presented in [5]).

The motional sense currents of both the right and left sense modes were fed to trans-impedance amplifiers and converted to voltages with $100 \mathrm{k} \Omega$ gain and demodulated to produce DC 


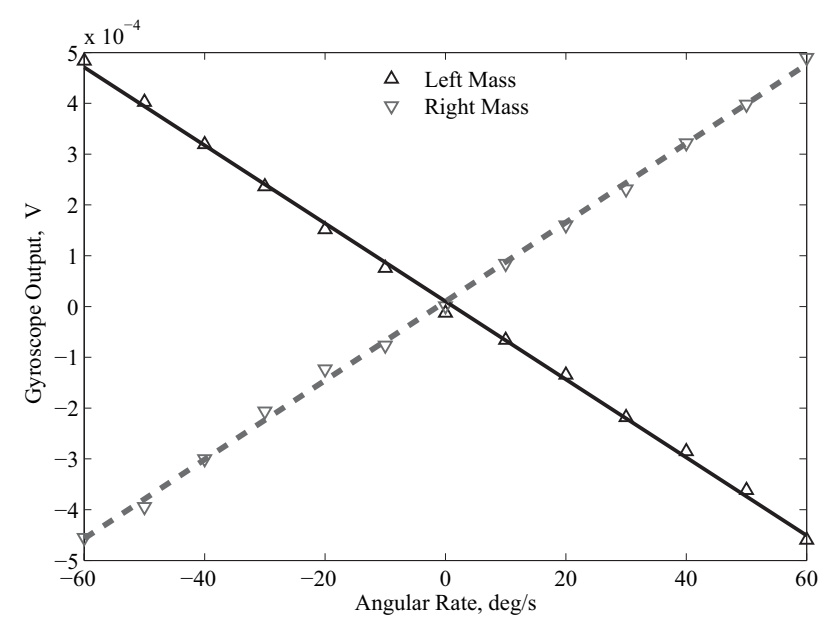

Fig. 6. Constant rate response of both right and left masses of the MDTF gyroscope

output voltages, see Fig. 6. The rate responses of the masses were in anti-phase, as expected. A linear fit was performed resulting in a measured sensitivity of $-7.68 \mu \mathrm{V} / \mathrm{deg} / \mathrm{s}$, zero offset of $10 \mu \mathrm{V}$, and linearity of $2.2 \%$ FSO for the left mass and a measured sensitivity of $7.78 \mu \mathrm{V} / \mathrm{deg} / \mathrm{s}$, zero offset of 10 $\mu \mathrm{V}$, and linearity of $2.2 \%$ FSO for the right mass.

\section{In-Operation Common Mode Rejection}

Finally, the multi-DOF tuning fork gyroscope operating at zero rate was subject to an impulse. The device was driven using the same drive voltage of $3.535 \mathrm{Vrms} A C$ at $2.167 \mathrm{kHz}$ combined with a $40 \mathrm{~V}$ DC bias to the central lateral comb drive, while an AC carrier voltage of $3 \mathrm{Vrms}$ at $18 \mathrm{kHz}$ was applied to the gyroscope mass. The motional sense currents were amplified and demodulated using EAM.

Fig. 7 (top) shows a time history of the demodulated amplitude for both the left and right mass for an applied impulse along the sense direction. The shock creates an output for both masses which would register as a false rate measurement. Fig. 7 (bottom) shows the differential signal resulting in a 14 $\mathrm{dB}$ reduction in amplitude.

\section{Conclusion}

This paper presents a demonstration of common mode rejection in the multi-DOF tuning fork MEMS rate gyroscope. The gain robustness of 2-DOF sense modes provides immunity to both environmental variations and fabrication imperfections, while the tuning fork driving architecture allows for common mode rejection by using differential signals since the resulting Coriolis responses will be in anti-phase.

A SOI MDTF prototype was characterized for both Coriolis and acceleration inputs. Constant angular rate experiments verified the anti-phase nature of the Coriolis motion resulting in sensitives of -7.68 and $7.78 \mu \mathrm{V} / \mathrm{deg} / \mathrm{s}$, zero offsets of $10 \mu \mathrm{V}$, and a linearity of $2.2 \%$ FSO (in a $60 \mathrm{deg} / \mathrm{s} \mathrm{range).} \mathrm{Impulse}$ responses demonstrated the common mode response of each sense system where a differential signal resulted in a $14 \mathrm{~dB}$
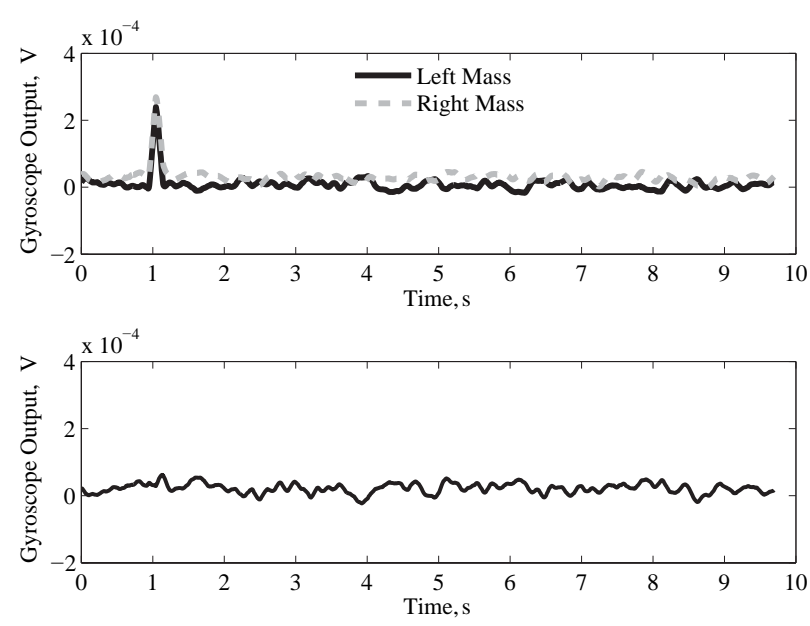

Fig. 7. Experimentally obtained time history of demodulated output for right and left masses due to shock input

reduction in demodulated amplitude versus the output of a single sense mode.

Thus, the MDTF design combines the previously demonstrated temperature and vibration robustness of multi-DOF sense modes with the common mode rejection capabilities of a tuning fork driving presented here. Our results show that the tuning fork architecture allows for further reduction of shock amplitude through differential signals while still maintaining an anti-phase Coriolis response.

\section{ACKNOWLEDGMENT}

This work was supported by BEI Technologies contract BEI-36974 and UC Discovery program ELE04-10202. The tested gyroscope was fabricated in the UCI Integrated Nanosystems Research Facility (INRF). Characterization of gyroscopes was carried out at the UCI MicroSystems Laboratory. The authors would like to acknowledge Lynn E. Costlow and Cenk Acar of BEI Technologies for the useful discussions. The authors would also like to thank John Porter of the Carl Zeiss Center of Excellence for use of the Ultra 55 SEM and $\mathrm{Vu}$ Phan of the UCI INRF for assistance with fabrication.

\section{REFERENCES}

[1] J. Geen, S. Sherman, J. Chang, and S. Lewis, "Single-chip surface micromachined integrated gyroscope with $50 \mathrm{deg} / \mathrm{h}$ allan deviation," IEEE J. Solid-State Circuits, vol. 37, no. 12, pp. 1860-1866, December 2002.

[2] C. Acar and A. Shkel, "Inherently robust micromachined gyroscopes with 2-DOF sense-mode oscillator," J. Microelectromech. Syst., vol. 15, no. 2, pp. 380-387, April 2006.

[3] M. Weinberg and A. Kourepenis, "Error sources in in-plane silicon tuningfork mems gyroscopes," J. Microelectromech. Syst., vol. 15, no. 3, pp. 479-491, June 2006.

[4] R. Neul, U. Gomez, K. Kehr, W. Bauer, J. Classen, C. Doring, E. Esch, S. Gotz, J. Hauer, B. Kuhlmann, C. Lang, M. Veith, and R. Willig, "Micromachined gyros for automotive applications," in Proc. IEEE Sensors 2005, Irvine, CA, USA, October 30-November 3, 2005.

[5] A. R. Schofield, A. A. Trusov, C. Acar, and A. M. Shkel, "Anti-phase driven rate gyroscope with multi-degree of freedom sense mode," in The 14th International Conference on Solid-State Sensors, Actuators and Microsystems (TRANSDUCERS '07), Lyon, France, June 10-14, 2007. 